\title{
COMPARATIVO DE RENDIMIENTO DE GRANO DE CINCO VARIEDADES DE SORGO GRANIFERO (Sorghum vulgare Pers) EN TERRENO DE ALTURA DE LA ZONA DE IQUITOS
}

Roger Riva Rodríguez

\section{RESUMEN}

El objetivo principal del presente trabajo fue determinar el comportamiento y rendimiento de cinco variedades de sorgo granífero en suelos de altura, con senillas procedentes del Programa de Selección de Sorgos para Suelos Á cidos, del CIAT-Colombia.

El experimento se desarrolló en terrenos del Centro de Investigaciones de "A hlpahuayo" del Instituto de Investigaciones de la A mazonía Peruana, 1IAP, que se encuentran ubicados en la margen derecha de la carretera I quitos - Nauta $\mathrm{K}$ m. 20. El suelo donde se realizó el experimento, corresponde a terraza alta; de textura franco; pH 4.5; bajos contenidos en materia orgánica $(0.67 \%)$, en fósforo (2 ppm), potasio $(0.16 \mathrm{meq} / 100 \mathrm{~g})$ y con alta saturación de aluminio $(70 \%)$.

Los tratamientos en estudio fueron las variedades IIA P 3-59; 4-1; 4-9; 534; 9-46; los mismos que fueron instalados mediante el diseño de bloque completamente al azar. Los resultados fueron IIAP 3-59; 4-9; 5-34; 9-46; 4-1; con rendimientos de $722.23 ; 663.75 ; 640.36 ; 511.70 ; 485.38 \mathrm{~kg} / \mathrm{ha}$. De grano respectivamente.

\section{SUM MARY}

The principal aim of this work was to set up the behavior and productiveness of five kinds of Granifeous Sorghum in high lands, using the seeds from the Selec-tion Program of Sorghum to A cid Soils, at CIA T-Colombia. The experiment was made on lands from the Center of Research "Allpahuayo" at the Peruvian A mazon Research Institute - IIAP. The Centre is found at the right side, $\mathrm{Km} 20$ of the Iquitos- $N$ auta roadway. The land, were the experiment was made, belon to high grounds; equal texture; $\mathrm{pH} \mathrm{4,5;} \mathrm{low} \mathrm{content} \mathrm{of} \mathrm{organic}$ subtance $(0.67 \%)$, phosfore $(2 \mathrm{ppm})$; potassium $(0.16 \mathrm{meq} / 100 \mathrm{~g})$ and with high aluminiun saturation (70\%).

Instituto de Investigaciones de la A mazonía Peruana- IIAP. A v. A belardo Quiñones K m. 2.5. A pto. 784 Iquitos- Perú. 
The treatment in study were the kinds; IIAP $3-59 ; 4-1 ; 4-9 ; 5-34 ; 9-46$; wich were set up by means of the Randimized Complete Range Design. The outcome was; IIAP 3-59; 4-9; 5-34; 9-41; 4-1; with productiveness of 722.23; $663.75 ; 640.36 ; 511.70$; of and $485.38 \mathrm{~kg} / \mathrm{ha}$ of grain, respectively.

Palabras claves : $\quad$ Sorghurn vulgare, rendimiendo en grano, tierra de altura.

\section{INTRODUCCION}

Según Ochse (1980) el Sorgo, (Sorghum vulgare Pers.), tuvo su origen en zonas tropicales; pero en la actualidad se cultiva en muchas de las zonas tropicales y templadas. El sorgo es utilizado para diferentes propósitos entre ellos está el sorgo granífero, que forma parte de la alimentación animal por ser fuente principal de carbohidrato y en parte de proteínas y vitaminas.

Los suelos de la llanura amazónica están representados por dos zonas bien definidas; la primera por una Llanura fluvial que sufre inundaciones periódicas que renueva sus nutrientes fertilizándose naturalmente y la segunda, Llanura aluvial no inundable que se caracteriza por presentar un cuadro de fertilidad relativamente baja, por la razón de estar sometida a elevadas temperaturas y excesiva precipitación, que contribuye a un alto grado de lexiviación, consecuencia de ello es su fuerte acidez, baja capacidad de intercambio catiónico y alto porcentaje de saturación de aluminio.

En la amazonía peruana, las áreas cultivadas de cereales como alimento son reducidas, que no satisface la demanda que exige la explotación ganadera; por este motivo, el Instituto de Investigaciones de la A mazonía Peruana (IIAP), ha participado en una evaluación de líneas de sorgo para suelos ácidos, con semillas procedentes del CIAT-Colombia.

En la necesidad de buscar una alternativa para mejorar la producción de granos, corno fuente de carbohidratos para la industria de alimentos balanceados para la producción animal, este trabajo trató de evaluar el comportamiento y rendimiento de cinco variedades de sorgo granífero en terrazas de altura. Estas variedades de sorgo granífero para su mejor conocimiento se les ha designado anteponiendo el nombre del IIAP.

\section{MATERIALES Y METODOS}

El presente trabajo experimental, se llevó a cabo en terrenos del Centro de Investigación de "Allpahuayo", propiedad del Instituto de Investigaciones de la 
A mazonía Peruana IIAP, ubicado en la margen derecha de la Carretera IquitosNauta, a la altura del km. 20 y a una altitud de 122.4 m.s.n.m. Según Holdrige(1967), el área es

clasificada ecológicamente como bosque húmedo tropical, con una temperatura media anual de $26.90 \mathrm{C}$ y una precipitación promedio anual de $2,457 \mathrm{~mm}$.

El suelo donde se realizó el trabajo corresponde al orden Inceptisol según el sistema americano de clasificación. La topografía es ligeramente inclinada; presentando una reacción muy fuertemente ácida, de textura franco; bajo contenido de materia orgánica $(0.61 \%)$, de fósforo $(2 \mathrm{ppm})$ y potasio $(0.16$ meq/100 g) y alta saturación de aluminio (70\%). El terreno corresponde una purma.

Las cinco (5) variedades que han sido estudiadas fueron las siguientes: IIAP 3-59; 4-1; 4-9; 5-34; 9-46.

El diseño experimental utilizado en este campo fue el de bloque completo al azar, con cuatro repeticiones.

EI análisis de varianza fue realizada por la prueba de $\mathrm{F}$ y la comparación de las medias por la prueba de DUNCAN a un nivel de significación de $5 \%$ de probabilidad.

La preparación de terreno se inició con la fase de rozo y quema; luego se procedió a la eliminación de tocones; la siembra se realizó el 12 de abril de 1985; empleándose el tacarpo y colocando 6 semillas por golpe en hilera que, previamente fueron tratados con aldrín al $2.5 \%$ polvo seco, utilizándose un distanciamiento de $0.60 \mathrm{~m}$ entre plantas y $0.25 \mathrm{~m}$ entre golpe, la resiembra se realizó al tercer día de la germinación de las semillas, y el desahije a los veinte días, dejando tres plantas por golpe.

Durante la conducción del experimento se registraron las siguientes enfermedades en las hojas y en las panojas : "Tizón de la hoja" (Helmistosporium sp), "Antracnosis" (Glomerella sp.), "M ancha zonada" (Glococercospora sp.) y las siguientes plagas "Cogollero" (Spodoptera frugiperda), y "Gusano Cañero" (Diatrea saccaralis); estas plagas y principalmente las enfermedades por su incidencia fueron determinadas de consideración económica.

\section{RESULTADOS Y DISCUSION}

La Tabla 1 esta referida a los tratamientos en estudio y los siguientes cuadros a los análisis de variancia y a la prueba de F. A sí mismo para comparar parejas de promedios de tratamientos se efectuó la prueba múltiple de DUNCAN. 
TABLA 1. Tratamiento de estudio

\begin{tabular}{|c|c|}
\hline CLAVE & VARIEDADES \\
\hline T1 & IIAP 3-59 \\
T2 & IIAP 4-1 \\
T3 & IIAP $4-9$ \\
T4 & IIAP 5-34 \\
T5 & IIAP 9-64 \\
\hline
\end{tabular}

TABL A 2. Análisis de variación para rendimiento de grano de cinco variedades de sorgo granífero (gramos/parcela)

\begin{tabular}{|l|l|l|l|l|}
\hline F. de V & G.L & C.M & F.C & F.0.05 \\
\hline Bloques & 3 & $60,113.33$ & 1.07 & 3.49 \\
Tratamientos & 4 & $30,305.00$ & 0.54 & 3.26 \\
Error & 12 & $56,005.00$ & & \\
\hline Total & 19 & & & \\
\hline
\end{tabular}

TABLA 3. Rendimiento promedio de cinco variedades de sorgo granifero ( $\mathrm{Kg} / \mathrm{ha}$ ) Resultado de la prueba de DUNCAN

\begin{tabular}{|c|c|c|}
\hline ORDEN DE M & VARIEDADES & RDTO. K g/ha \\
\hline 1 & IIAP 3-59 & 722.23 \\
2 & IIAP 4-9 & 663.75 \\
3 & IIAP 5-34 & 640.36 \\
4 & IIAP 9-46 & 511.70 \\
5 & IIAP 4-1 & 485.38 \\
\hline
\end{tabular}

* $\quad$ Promedios unidos por una misma línea no muestra diferencia significativa.

TABL A 4. Análisis de variancia por altura de planta de cinco variedades sorgo granifer 0 . (m)

\begin{tabular}{|l|l|l|l|l|}
\hline F. de V. & G.L & C.M. & F.C. & F.0.05 \\
\hline Bloques & 3 & 0.04 & 1.33 & 3.49 \\
Tratamientos & 4 & 0.01 & 0.33 & 3.26 \\
Error & 12 & 0.03 & & \\
\hline Total & 19 & & & \\
\hline
\end{tabular}


TABLA 5. Altura de la planta promedio de cinco variedades de sorgo granifero. (m) Resultados de la prueba de DUNCAN

\begin{tabular}{|c|c|c|}
\hline ORDEN DE M & VARIEDADES & ALT. PLANTA (m) \\
\hline 1 & IIAP 5-34 & 1.62 \\
2 & IIAP 4-9 & 1.53 \\
3 & IIAP 9-46 & 1.51 \\
4 & IIAP 4-1 & 1.49 \\
5 & IIAP 3-59 & 1.47 \\
\hline
\end{tabular}

TABL A 6. A nálisis de V ariancia por longitud de panoja de cinco variedades de sorgo granífero. $(\mathrm{cm})$

\begin{tabular}{|l|l|l|l|l|}
\hline F. de V. & G.L & C.M. & F.C. & F.0.05 \\
\hline Bloques & 3 & 3.05 & 2.01 & 3.49 \\
Tratamientos & 4 & 5.12 & 3.37 & 3.26 \\
Error & 12 & 1.52 & & \\
\hline Total & 19 & & & \\
\hline
\end{tabular}

* Significativo al 0.05

TABL A 7. L ongitud de panoja promedio de cinco variedades de sorgo granifero. (cm) Resultado de la prueba de DUNCAN

\begin{tabular}{|c|c|c|}
\hline ORDEN DE M. & VARIEDADES & LONG.DE PAN OJA $(\mathrm{cm})$ \\
\hline 1 & IIAP 3-59 & 18.12 \\
2 & IIAP 4-1 & 16.22 \\
3 & IIAP 9-46 & 15.93 \\
4 & IIAP 5-34 & 15.36 \\
5 & IIAP 4-9 & 15.17 \\
\hline
\end{tabular}


TABLA 8. Análisis de Variancia para peso de panoja de cinco variedades sorgo granifero. (g)

\begin{tabular}{|l|l|l|l|l|}
\hline F. de V. & G.L & C.M. & F.C. & F.0.05 \\
\hline Bloques & 3 & 2,085 & 0.77 & 3.49 \\
Tratamientos & 4 & 1,630 & 0.60 & 3.26 \\
Error & 12 & 1,710 & & \\
\hline Total & 19 & & & \\
\hline
\end{tabular}

TABLA 9. Peso de panoja promedio de cinco variedades de sorgo granifero, (g). Resultado de prueba de DUNCAN.

\begin{tabular}{|c|c|c|}
\hline ORDEN DE M. & VARIEDADES & $\begin{array}{c}\text { LONG.DE PAN OJA (cm) } \\
\text { Peso de panoja }(\mathrm{g})\end{array}$ \\
\hline 1 & IIAP 3-59 & 210.0 \\
2 & IIAP 4-9 & 200.02 \\
3 & IIAP 5-34 & 185.0 \\
4 & IIAP 9.46 & 172.5 \\
5 & IIAP 4-1 & 160.0 \\
\hline
\end{tabular}

\subsection{Rendimiento de la planta}

Haciendo un análisis de los rendimientos de las variedades de sorgo granífero de acuerdo con la prueba Duncan realizada a un nivel de 0.05 de significancia, no se encontró diferencia significativa entre promedios de tratamiento; (Ver Tabla 2).

En la Tabla 3 se puede apreciar que la variedad IIA P 3-59 fue el que obtuvo el mejor rendimiento con $722.23 \mathrm{~kg}$./ha. Correspondiendo el más bajo rendimiento a la Variedad IIAP $4-1$ con $485.38 \mathrm{~kg} . \mathrm{Ha}$, ubicándose las demás variedades entre estos extremos.

Los rendimientos como se pueden apreciar son muy bajos, comparados con los rendimientos obtenidos por Churango (1975) con las variedades de sorgo granífero IPB/EW-1 10; IPB/ML-135; 72025 con $4,025,3,066$ y $2,944 \mathrm{~kg} / \mathrm{ha}$ de grano respectivamente cultivados en suelos de altura de textura franco-arcillo-arenoso de Zungaro Cocha, centro experimental de la U niversidad Nacional de la 
A mazonía Peruana. Los bajos rendimientos obtenidos se deben al uso del suelo en condiciones naturales sin la adición de ningún tipo de fertilizante ni

enmienda; en cambio los resultados obtenidos por Churango (1975), se pueden considerar significativos, teniendo en cuenta la incorporación de gallinaza como abono en una proporción de 10 t/ha. Es obvio indicar que las gramíneas son muy exigentes en suelos fértiles.

\subsection{Altura de la planta}

Con respecto a la altura y de acuerdo a la prueba de Duncan a un nivel de 0.05 ; no se encontró diferencia significativa entre los tratamientos estudiados tal como se puede ver en la Tabla 5 . También se observó que los tallos fueron muy delgados y débiles debido a la deficiencia de nutrientes del suelo, especialmente fósforo, sobre el particular Sánchez (1974) manifiesta que en los suelos deficientes en fósforo, las plantas presentan desarrollo lento sobre todo en las raíces, produciéndose tallos delgados y alargados. Mientras tanto Gispert (1983), refiriéndose a la acidez, indica que un suelo de pH ácido, produce alteraciones morfológicas en las plantas; así como raíces raquíticas, hojas de aspecto purpúreo y rígido y no se desarrolla el tallo.

\subsection{Longitud de panoja}

La prueba de Duncan para la longitud de panoja nos indica que existe diferencia significativa entre los tratamientos estudiados, tal como se observa en las Tablas 6 y 7. La variedad 1IAP 3-59 es significativa sobre las variedades IJ A P 9-46, 5-34, 4-9; pero no es significativa con la variedad IIAP 4-1. Entre las variedades IIAP 4-1, 9-46, 5-34 y 4-9 no se encontró diferencia significativa. Esta característica de longitud de panoja, comparado con los resultados obtenidos por Churango (1975) y Correa (1967), que alcazaron promedios de 23.28 y $18.87 \mathrm{~cm}$ respectivamente son mayores que los obtenidos en el presente trabajo que alcanzó un promedio de $16.27 \mathrm{~cm}$ debiéndose a fuentes antes expuestas. Es importante indicar que Correa (1967), trabajó en suelos de Ilanura fluvial reciente. 


\subsection{Peso de panoja}

En lo que se refiere al peso de panoja, no se encontró diferencia significativa entre los tratamientos de acuerdo a la prueba de Duncan, tal como se observa en las Tablas 8 y 9; a pesar de ello, los rendimientos obtenidos son bajos, siendo inferiores comparados con los obtenidos por Díaz (1972), con las variedades de sorgo granífero en soca NK-300, IPB/M L-135 y IPB/M L-136 con 1,407.4 1, 1,203.70 y 1,009.26

$\mathrm{kg} / \mathrm{ha}$ de grano, que trabajo también en suelos de llanura fluvial reciente. Al respecto Garman (1975) manifiesta que el trabajar en suelos pobres en nutrientes especialmente en fósforo, influye en el bajo rendimiento de granos frutos y semillas.

\section{CONCLUSIONES}

4.1 La variedad IIAP 3-59 obtuvo un mejor rendimiento, con $722.23 \mathrm{~kg} / \mathrm{ha}$ de grano, pero no fue estadísticamente significativa sobre las demás variedades.

4.2 Los rendimientos obtenidos fueron notoriamente bajos debido a la pobreza en nutrientes, acidez del suelo, etc.

4.3 Las características agronómicas de las variedades en estudio fueron poco deseables bajo estas condiciones de trabajo, debido a las fuentes antes expuestas. 


\section{BIBLIOGRAFIA}

CORREA DEL A., 1967. Efecto de la densidad de siembra en el rendimiento de sorgo de grano en la zona de Iquitos. Tesis Ing0 Agr. UNAP. Iquitos Perú. $76 \mathrm{p}$.

CHURANGO C., 1975. Comparativo de 11 variedades de sorgo granífero en la zona alta de Iquitos. Tesis Ing0 A gr. UNAP. Iquitos - Perú. 52 p.

DIA Z J., 1972. Evaluación del rendimiento de sorgo granífero en soca en la zona de Iquitos. Tesis Ing0 A gr. UNAP. Iquitos - Perú. 49 p.

GARM AN H., 1975. M anual de fertilizantes Editorial Limusa M \& ico 292 p.

GISPERT C., 1983. Biblioteca práctica agrícola y ganadera. Fundamentos de agricultura. Impreso en artes gráficas Grijalbo S.A. España. 204 p.

HOLDRIGE, L.E., 1967. Zonas ecológicas de vida tropical. Science Center San J osé, Costa Rica. $200 \mathrm{p}$.

OCHSE J.J., 1980. Cultivo y mejoramiento de plantas tropicales y sub tropicales, volumen 11, Edit. Limusa; impreso en Mexico (pag. 8551536).

PALMAN CH, E., 1981. Desarrollo y control de enfermedades de las plantas. Editorial Limusa M exico V.I. $223 \mathrm{p}$.

SANCHEZ G.L., 1974. Guía del agricultor Edit. A edos Barcelona 2da. Edición $408 \mathrm{p}$. 